\section{Cistitis enfisematosa: una inusual forma de presentación de la infección del tracto urinario bajo}

\author{
NICOLÁS VERA A. ${ }^{a}$, CHRISTIAN ZWANZGER M. ${ }^{b}$, PABLO TRONCOSO C.
}

\section{Emphysematous cystitis. Report of one case}

Emphysematous cystitis is found in diabetic patients and in individuals with urinary stasis and immunosuppression. We report a 58-year-old male with hypertension, type 2 Diabetes on insulin treatment and central nervous system vasculitis on immunosuppressive therapy. He was admitted with weight loss and gait instability. A PET-CT showed a circumscribed image of air in the bladder contour without involving the upper urinary tract, suggesting emphysematous cystitis. Re-interrogated, the patient referred pneumaturia, dysuria and febrile sensation one week before admission. Urine culture showed Enterobacter aerogenes. He was treated with a urinary catheter, metabolic control and parenteral antimicrobials. The patient was discharged without symptoms 21 days after admission, with the bladder catheter.

(Rev Med Chile 2015; 143: 387-390)

Key words: Cystitis; Enterobacter Aerogenes, Urinary Tract Infections.

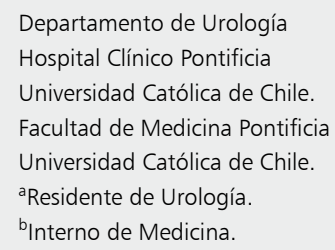

Recibido el 1 de octubre de 2013 aceptado el 22 de diciembre de 2014.

Correspondencia a:

Nicolás Vera A.

Departamento de Urología

Hospital Clínico Pontificia

Universidad Católica de Chile.

Marcoleta 367, Santiago, RM,

Chile.

Teléfono: 23543468

nicolasveraar@gmail.com
Lim a cistitis enfisematosa es una entidad aislada y muy poco frecuente, que se caracteriza por la presencia de gas en la pared de la vejiga y ocasionalmente, dentro de ella. Está asociada, en general, a pacientes de sexo femenino, diabéticos, inmunosuprimidos, con infección urinaria recurrente y ectasia urinaria ${ }^{1,2}$. Hasta la fecha, existen escasas publicaciones en la literatura internacional y ninguna a nivel nacional respecto a esta enfermedad. El objetivo de esta presentación es describir un caso clínico y evaluar aspectos diagnósticos y terapéuticos relacionados a esta patología.

\section{Caso clínico}

Se presenta el caso de un paciente de sexo masculino, 58 años, con antecedentes de hipertensión arterial, diabetes mellitus tipo 2 en tratamiento con insulina y vasculitis del sistema nervioso central de reciente diagnóstico, en tratamiento inmunosupresor con prednisona y azatioprina.

Ingresó por cuadro de baja de peso de $10 \mathrm{~kg}$ en 1 mes, compromiso del estado general e inestabilidad de la marcha, con compromiso motor y sensitivo de extremidades inferiores. Dentro del estudio de este cuadro se realizó PET-CT de cuerpo entero con el fin de descartar causa paraneoplásica, el cual evidenció imagen de aire que circunscribía aisladamente el contorno vesical, sin comprometer la vía urinaria alta, compatible con cistitis enfisematosa (Figuras 1 y 2 ).

$\mathrm{Al}$ interrogatorio dirigido el paciente refería presentar pneumaturia, disuria, polaquiuria, tenesmo vesical, pujo y sensación febril una semana previa al ingreso, sin calofríos. Sin hospitalización reciente ni instrumentalización de la vía urinaria. Sedimento de orina mostró leucocituria $>50 \mathrm{~GB} /$ campo, sin hematuria. El urocultivo (VITEK 2) fue positivo para Enterobacter aerogenes (resistente a cefalosporinas) y los parámetros inflamatorios eran normales (PCR $<1 \mathrm{mg} / \mathrm{dL}$, VHS $5 \mathrm{~mm}$, leucocitos en sangre 7.900, sin elementos inmaduros en sangre periférica). Función renal normal (creatininemia 0,89 mg/dL, VFG $>60 \mathrm{ml} / \mathrm{min}$ ). El paciente ingresó en buenas condiciones generales, 


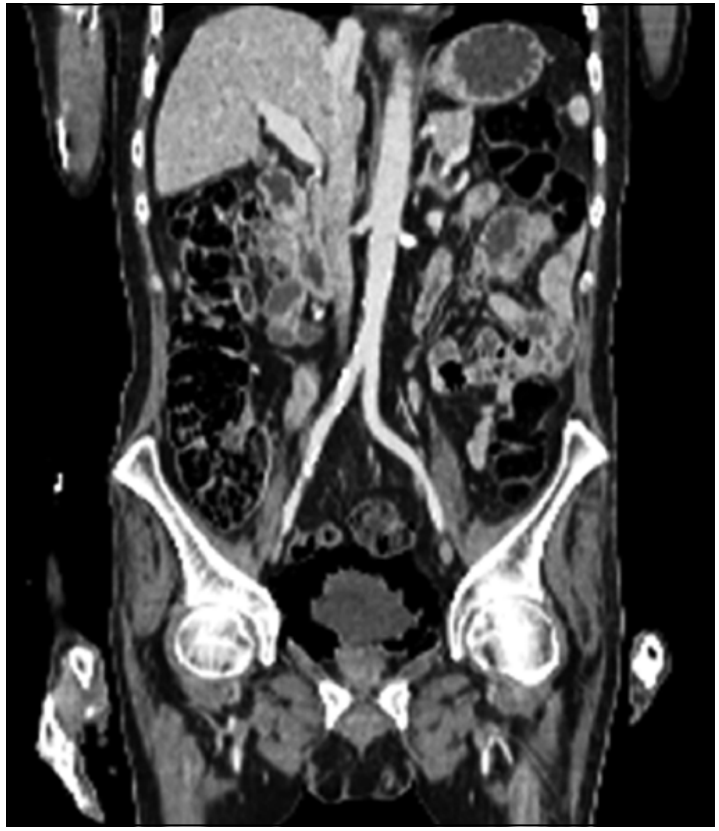

Figura 1. PET SCAN Visión frontal.

hemodinámicamente estable y afebril. Se indicó cateterismo vesical, control metabólico estricto con insulina y tratamiento antibiótico parenteral con imipenem, considerando antibiograma, condición del paciente y estado de inmunosupresión, completando 14 días de tratamiento. Evolucionó favorablemente, afebril, sin compromiso hemodinámico ni alteración significativa de parámetros sépticos. Fue dado de alta a los 21 días de hospitalización, asintomático, manteniendo catéter vesical y con imagen de aire vesical en regresión en radiografía simple de control.

El paciente acudió a control al mes del alta en buenas condiciones generales, sin síntomas urinarios. Se solicita radiografía renal y vesical simple (Figura 3), donde se evidenció regresión completa de los signos de cistitis enfisematosa, por lo que se decidió retiro de la sonda vesical.

\section{Discusión}

La presentación clínica habitual de la cistitis enfisematosa es similar a la de una infección urinaria, incluyendo disuria, polaquiuria y hematuria, aunque en algunos casos puede presentarse pneu-

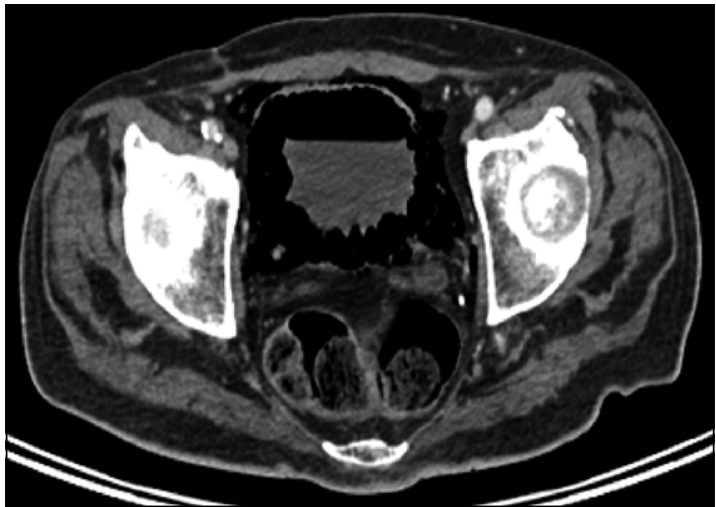

Figura 2. PET SCAN Visión transversal.

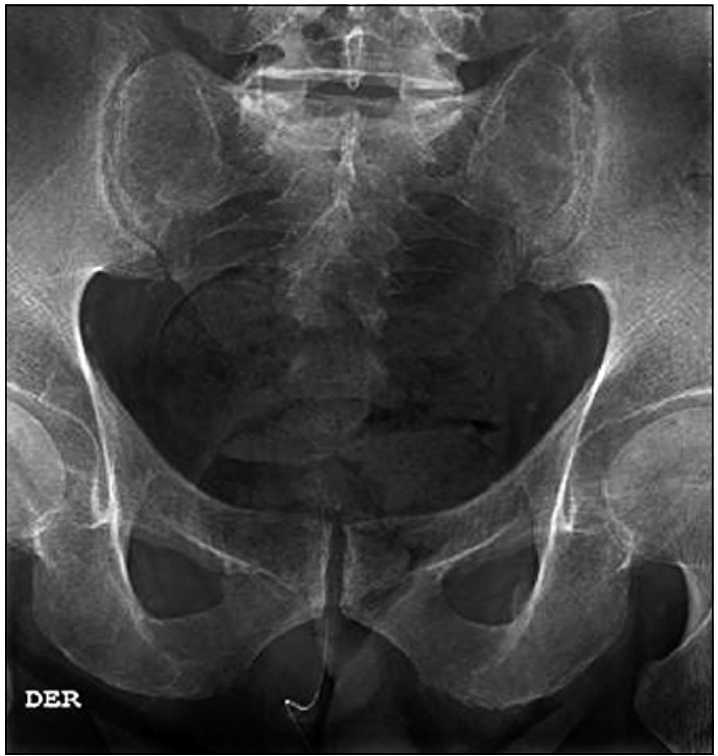

Figura 3. Radiografía Vesical Simple.

maturia ${ }^{3}$. En otros casos puede manifestarse como dolor hipogástrico, macrohematuria o molestias urinarias inespecíficas. Sin embargo, la forma de presentación puede ser variable y atípica, desde pacientes asintomáticos hasta casos potencialmente letales, sin una estricta correlación entre el grado de inflamación y estado clínico del paciente ${ }^{4,5}$. Por esta razón se requiere un alto índice de sospecha ${ }^{6}$ y una revisión dirigida del estudio radiológico, en particular, en pacientes de alto riesgo ${ }^{7}$.

Dentro de su fisiopatología jugarían un rol crucial la mayor disponibilidad de glucosa a nivel 
tisular y la disfunción del sistema inmune. Ambos fenómenos favorecen la proliferación de bacterias con capacidad de fermentación, las cuales a través de este proceso producirían $\mathrm{CO}_{2}^{8}$. Las bacterias obtienen energía a través de la vía glucolítica anaerobia, por la fermentación de la glucosa, obteniendo ATP y $\mathrm{NAD}^{+}$que se transforman en $\mathrm{NADH}$ y piruvato. Algunos microorganismos como E. coli, convierten el ácido fórmico a $\mathrm{CO}_{2} \mathrm{e}$, $\mathrm{H}_{2}$. Las burbujas formadas contienen nitrógeno, oxígeno, dióxido de carbono, hidrógeno e incluso amonio y metano. Dependiendo si las burbujas están sometidas a presiones positivas o negativas se quedan en el lumen del órgano o pasan a la pared del mismo e incluso al torrente sanguíneo. El daño tisular por el gas y la microangiopatía causan acumulación de gas, que se ve potenciado por el déficit inmunitario y el mal control metabólico ${ }^{8,9}$.

Como agentes etiológicos encontramos principalmente gérmenes bacilos Gram negativos aerobios, siendo el más frecuente $E$. Coli ${ }^{10}$. Otros microorganismos son Klebsiella pneumoniae, Proteus mirabilis, Staphylococcus aureus, Enterobacter aerogenes, Streptococcus sp. y Nocardia. Hay descritos casos esporádicos por hongos Candida albicans ${ }^{11}$ y Candida tropicallis y anaerobios como Clostridium perfringens ${ }^{12}$.

La presencia de gas en la infección del tracto urinario no es exclusiva de la vejiga y ha sido descrito con mayor frecuencia en pielonefritis del paciente diabético, comprometiendo vía urinaria alta y parénquima renal ${ }^{1}$.

El diagnóstico de esta condición generalmente se realiza por métodos de imagen ${ }^{7}$, pudiendo ser sospechado por imágenes simples como radiografía simple o ecografía, pero fundamentalmente tomografía computada ${ }^{13}$, que permite la detección temprana de aire vesical intramural-intraluminal, así como la demostración de otras causas de gas vesical o intrapelviano y detección de posibles complicaciones $^{14,15}$.

El tratamiento estándar consiste en drenaje vesical para reducir la presión intravesical, control glicémico estricto y antibioterapia parenteral de amplio espectro ajustando según antibiograma ${ }^{16}$. El pronóstico suele ser favorable ${ }^{17}$, sin embargo, se pueden presentar complicaciones como sepsis persistente, compromiso de vía urinaria alta $y$, hasta en $20 \%$ de los casos pueden requerir una intervención quirúrgica, asociándose a mayor morbimortalidad $^{18}$.
En el caso presentado, el diagnóstico y tratamiento precoz, probablemente, influyó en la buena evolución. Aunque no existen reportes de recurrencia de esta forma inusual de presentación de la infección del tracto urinario inferior es probable que mientras persistan las condiciones predisponentes asociadas, el riesgo también será mayor.

\section{Referencias}

1. Patel NP, Lavengood RW, Fernandes M, Ward JN, Walzak MP. Gas-forming infections in genitourinary tract. J Urol 1992; 39: 341-5.

2. Quint HJ, Drach GW, Rappaport WD, Hoffmann CJ. Emphysematous cystitis: a review of the spectrum of disease. J Urol 1992; 147: 134-7.

3. Ho KMT, Sole M. Pneumaturia due to gas-producing E. coli and urinary stasis. Br J Urol 1994; 73 (5): 588-9.

4. Joshi N, Caputo G, Weitekamp MR, Karchmer AW. Infections in patients with diabetes mellitus. N Engl J Med 1999; 341 (25): 1906-12.

5. Mokabberi R, Ravakhab K. Emphysematous urinary tract infections. Am J Med Sci 2007; 333 (2): 111-6.

6. Selley JK, Kane BG, Kane KE. Importance of obtaining a detailed medical history in diagnosing emphysematous cystitis. J Am Osteopath Assoc 2010; 110: 91-4.

7. Bobba RK, Arsura EL, Sarna PS, Sawh AK. Emphysematous cystitis: an unusual disease of the genito-urinary system suspected on imaging. Ann Clin Microbiol Antimicrob 2004; 3: 30.

8. Yang WH, Shen NC. Gas-forming infection of the urinary tract: an investigation of fermentation as a mechanism. J Urol 1990; 143: 960-4.

9. Huang JJ, Chen KW, RUAN MK. Mixed acid fermentation of glucose as a mechanism of emphysematous urinary tract infection. J Urol 1991, 146: 148-51.

10. Grupper M, Kravtsov A, Potasman I. Emphysematous Cistitis. Illustrative case report and review of the literature. Medicine (Baltimore) 2007; 86: 47-53.

11. Bartkowski DP, Lanesky JR. Emphysematous prostatitis and cystitis secondary to Candida albicans. J Urol 1988; 139: 1063-5.

12. West TE, Holley HP, Lauer AD. Emphysematous cystitis due to Clostridium perfringens. JAMA 1981; 246: 363-4.

13. Asada S, Kawasaki T. Images in clinical medicine. Emphysematous cystitis. N Engl J Med 2003; 17: 258.

14. Young YR, Sheu BF, Lee CC, Chang SS, Li PL, Wu YS. Images in emergency medicine. Emphysematous cystitis. Ann Emerg Med 2008; 51 (3): 230-61. 
15. Bailey H. Cystitis emphysematosa: 19 cases with intraluminal and interstitial collections of gas. Am J Roentgenol Radium Ther Nucl Med 1961; 86: 850-62.

16. Thomas AA, Lane BR, Thomas AZ, Remer EM, Campbell SC, Shoskes DA. Emphysematous cystitis: a review of 135 cases. BJU Int 2007; 100: 17-20.

17. Bjurlin MA, Hurley S, Kim DY, Cohn MR, Jordan MD,
Kim R, et al. Clinical outcomes on nonoperative management in emphysematous urinary tract infections. Urology 2012; 79 (6): 1281-5.

18. Bañón Pérez VJ, García Hernández JA, Valdelvira Nadal P, Nicolás Torralba JA, Server Pastor G, Coves R, et al. Intraperitoneal bladder perforation in emphysematous cystitis. Actas Urol Esp 2000; 24: 501-3. 